\title{
Article
}

http://dx.doi.org/10.11646/phytotaxa.288.3.11

\section{Vernonanthura nana (Asteraceae: Vernonieae), a new species from the Paraná State (Brazil)}

\author{
ALVARO JOSE VEGA ${ }^{1,2^{*}} \&$ MASSIMILIANO DEMATTEIS ${ }^{1,2}$ \\ IInstituto de Botánica del Nordeste (UNNE-CONICET), Casilla de Correo 209, Código Postal 3400, Corrientes, Argentina. \\ ${ }^{2}$ Facultad de Ciencias Exactas y Naturales y Agrimensura, Universidad Nacional del Nordeste, Argentina. \\ *Corresponding author; email: alvarojose_82@yahoo.com.ar
}

\begin{abstract}
A new species of Vernonanthura (Asteraceae), V. nana, from the Brazilian state of Paraná is described and illustrated. The new species resembles $V$. squamulosa but differs in its geographical distribution, habitat, shape and pubescence of the leaves, and involucre length.
\end{abstract}

Key words: Brazil, Compositae, new species, Paraná

\section{Introduction}

Vernonieae Cassini (1819: 203) is one of the largest tribes in the Asteraceae comprising approximately 1700 species distributed across the tropical regions of Asia, Africa and America (Robinson 2007, Keeley \& Robinson 2009). Traditional classifications grouped the members of the tribe into six different subtribes based on inflorescence patterns, persistence of phyllaries, pollen morphology, chemical composition and chromosome numbers (Bremer 1994). However, recent morphological and phylogenetic studies based on molecular data (Keeley \& Robinson 2009) recognized a total of 21 subtribes, 15 from the New World and 6 of the Old World. The new classification proposed significant taxonomic changes; resulting from these studies was the reduction in the size and distribution of the core genus Vernonia Schreber (1791: 541) and the recognition of several new genera. All species of Vernonia were confined to the New World with the vast majority in continental North America (Robinson 1999). One of these newly segregated genera is Vernonanthura Robinson (1992: 65). The original description of Vernonanthura included 63 species (Robinson 1992), to which Robinson (1995) added seven more. Dematteis (2006) also described a new species, and more recently Vega \& Dematteis $(2010,2011$ a) transferred another five South American taxa from Vernonia to Vernonanthura.

Species of the genus are shrubs or small trees with thyrsoid to pyramidal inflorescences and individual cymose to corymbose branches. They are distributed from southern Mexico to central Argentina, but are mostly concentrated in southeastern Brazil (Robinson 1992). Almost all Vernonanthura taxa are diploid with 2n $=34$, excluding V. pinguis (Grisebach 1879: 165) Robinson (1992: 74), which constitutes the only tetraploid species of the genus with $2 \mathrm{n}=$ 68 (Dematteis \& Fernandez 1998; Dematteis 2002; Vega \& Dematteis 2012). Vernonanthura is characterized by tricolporate, subechinolophate pollen grains with a continuous microperforate tectum, and depressions delimited by irregular ridges (Vega \& Dematteis 2011b). The generic limits of Vernonanthura are clear and there are no major problems in its circumscription. However, due to the great diversity of its species in central and southeastern Brazil, there are many species that have not yet been described. In the present paper, a new species from the Brazilian state of Paraná is described, illustrated and compared with related species.

\section{Description of the new species}

Vernonanthura nana A. J. Vega \& Dematt., spec. nov. (Fig. 1)

Type:-BRAZIL. Paraná: Palmeira, 3 August 1980, L. T. Dombrowski 11783 (holotype MBM). 


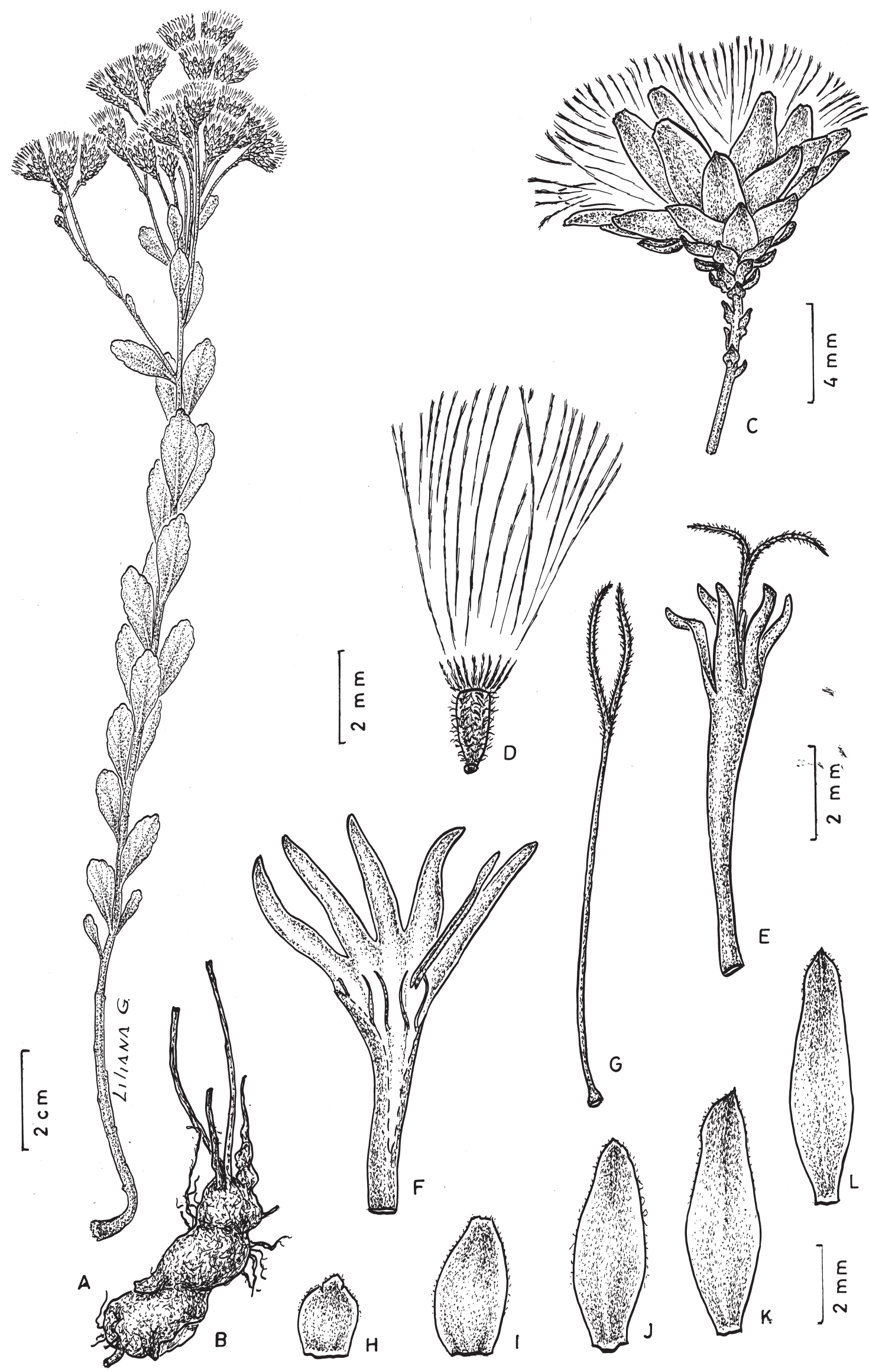

FIGURE 1. Vernonanthura nana A. J. Vega \& Dematt. A. Plant. B. Xylopodia. C. Capitulum. D. Cypsela. E. Floret, showing style. F. Corolla, desiccated, showing anthers. G. Style. H-I. Outer phyllary. J. Middle phyllary. K-L. Inner phyllaries. 
Erect subshrub up to $50 \mathrm{~cm}$ high, with small rounded xylopodia, from which one or several stems are risen, striate, loosely pilose to glabrous, laxly leafy up to inflorescence, $2-4 \mathrm{~mm}$ in diameter. Lower leaves deciduous, middle and upper leaves persistent, sessile, coriaceous. Leaf blades obovate to obovate-oblong, $0.6-3.5 \mathrm{~cm}$ long, $0.4-1.5 \mathrm{~cm}$ wide, margins crenate to serrulate from middle to apex, apically rounded to acute, attenuate at base, glandular dotted on both faces. Inflorescence corymbose to paniculiform with individual branches corymbose, bearing 3-8(-10) heads. Bracts of inflorescence oblong-obovate, $2-8 \mathrm{~mm}$ long. Capitula pedunculate, peduncles $(0.3-) 0.5-1.7(-2) \mathrm{cm}$ long, pilose. Involucre turbinate, 6-8 mm long, 6-10 mm wide. Phyllaries in 9-11(-12) series, coriaceous, glabrous or ciliate at margin, inner phyllaries linear-lanceolate, 6-7 mm long, middle ones lanceolate, 4-5 mm long, outer ones ovatelanceolate, 3-4 series commonly extending onto the peduncles, $2-3 \mathrm{~mm}$ long. Florets violet. Corollas 9-12 $\mathrm{mm}$ long, lobes lanceolate, glandular, 3.5-4 mm long. Anthers glandular, 3-4 mm long, apical anther appendages lanceolate, 0.7 $\mathrm{mm}$ long. Styles with basal node, 9-11(-12) $\mathrm{mm}$ long, branches $3 \mathrm{~mm}$ long. Cypselas ribbed, pilose and glandular, 3-3.5 mm long. Pappus biseriate, inner bristles 5-6 mm long, outer scales $0.8-1.2 \mathrm{~mm}$ long.

Paratypes:-BRASIL. Paraná. Mun. Palmeiras, Colonia Witmarsum. 30 Jul 1961, G. Hatschbach 8162 (BR, MBM); Pinhaes, in paludosis, 1 Oct 1910, P. Dusén 10329 (G, P); Rio dos Papagaios, 22 Apr 1993, A. C. Cervi 4092 (MBM); Mun. Ponta Grosa, Furnas Gemeas, Passo do Pupo, 21 May 2011, M. G. Caxambu et al. 3405 (MBM); Mun. Balsa Nova, Ponte dos Arcos, 21 Jul 2005, C. Kozera and O. P. Kozera 2292 (MBM); Mun. Lapa, Sitio Santa Bernadete, 29 Jul 1959, Braga \& Lange s.n. (MBM). Mun: Campinas Grande do Sul, Serra do Ibitiraquire, subida ao Pico Paraná, 17 Aug 1996, O. S. Ribas et al. 1471 (MBM); Trilha para o Pico Paraná, 05 Oct 1997, J. M. Silva et al. 2047 (MBM); Pico Caratuva, 1950 m, 5 Oct 1967, G. Hatschbach \& J. G. Stutts 17321 (BC); Mun: Morretes, Serra Marumbé, subida ao Pico Olimpo, 23 Oct 1995, O. S. Ribas 915 (MBM); Mun: Antoninas, Usina hidrelétrica Parigot de Souza, cota 800, 17 Jul 1991, G. Hatschbach \& J. Saldanha 55657 (C, MBM). Jaguariaíva, 30 Jul 1994, J. Semir et al. 31934 (UEC).

In the treatment of Vernonia subsect. Chamaedrys by Stutts (1988), Vernonanthura crassa (Vellozo 1825: 305) Robinson (1992: 70), V. montevidensis (Sprengel 1826: 229) Robinson (1992: 72) and V. squamulosa (Hooker \& Arnott 1836: 44) Robinson (1992: 74) were placed within Vernonia ser. Nitidulae (Cabrera 1944: 347) Stutts (1988: 42). The new species can also be placed into this group because they share the arrangement of the inflorescence and have phyllaries extending onto the peduncles. However, the new species differs considerably from the others in its habit, shape and size of the involucre and leaf pubescence (Table I). Vernonanthura crassa is a shrub $120-150 \mathrm{~cm}$ high, with involucres urceolate-campanulate, $12-17 \mathrm{~mm}$ long, leaf blades ovate to narrowly elliptic, 3-6 $\mathrm{cm}$ long, $(0.5-) 1.5-2.5 \mathrm{~cm}$ wide, densely greyish to brownish, lanate beneath. Vernonanthura montevidensis is a shrub 80-300 $\mathrm{cm}$ high, with leaf blades oblong-lanceolate to lanceolate, $2-6 \mathrm{~cm}$ long, $0.3-1 \mathrm{~cm}$ wide, peduncles $0.1-1.2 \mathrm{~cm}$ long, and involucres 5-8 $\mathrm{mm}$ long.

TABLE 1. Comparison between the new species and closely related taxa.

\begin{tabular}{|c|c|c|c|c|}
\hline Character & V. nana & V. crassa & V. montevidensis & V. squamulosa \\
\hline Plant height $(\mathrm{cm})$ & $30-50$ & $120-150$ & $80-300$ & $100-300$ \\
\hline Leaf size $(\mathrm{cm})$ & $0.6-3.5 \times 0.4-1.5$ & $3-6 \times(0.5-) 1.5-2.5$ & $2-6 \times 0.3-1$ & $3-11 \times 1.5-3.5$ \\
\hline Leaf shape & $\begin{array}{l}\text { obovate to obovate- } \\
\text { oblong }\end{array}$ & $\begin{array}{l}\text { ovate to narrowly } \\
\text { elliptic }\end{array}$ & $\begin{array}{l}\text { oblong-lanceolate to } \\
\text { lanceolate }\end{array}$ & $\begin{array}{l}\text { lanceolate to } \\
\text { obovate-lanceolate }\end{array}$ \\
\hline Inflorescence branching & corymbose & corymbose & corymbose & corymbose \\
\hline Pedicel length (mm) & $5-17$ & $5-10$ & $1-12$ & $10-30$ \\
\hline Involucre shape & turbinate & urceolate-campanulate & turbinate & turbinate \\
\hline Involucre length (mm) & $6-8$ & $12-17$ & $5-8$ & $9-10$ \\
\hline
\end{tabular}

Most specimens of the new species have been commonly misidentified as $V$. squamulosa, which shows a clear morphological similarity. However, both species can be easily separated by their geographical distribution, habitat, shape and pubescence of the leaves, and involucre length. Vernonanthura squamulosa is distributed in western South America, from Bolivia to northwestern Argentina, while the new species is endemic to the Paraná state in Brazil (Fig. 2). Also, $V$. squamulosa is a shrub $100-300 \mathrm{~cm}$ tall, with leaf blades lanceolate to obovate-lanceolate, tomentose below, 3-11 cm long, $1.5-3.5 \mathrm{~cm}$ wide, involucre $0.9-1.0 \mathrm{~cm}$ long. The group of species related to $V$. nana can be distinguished by the following key. 


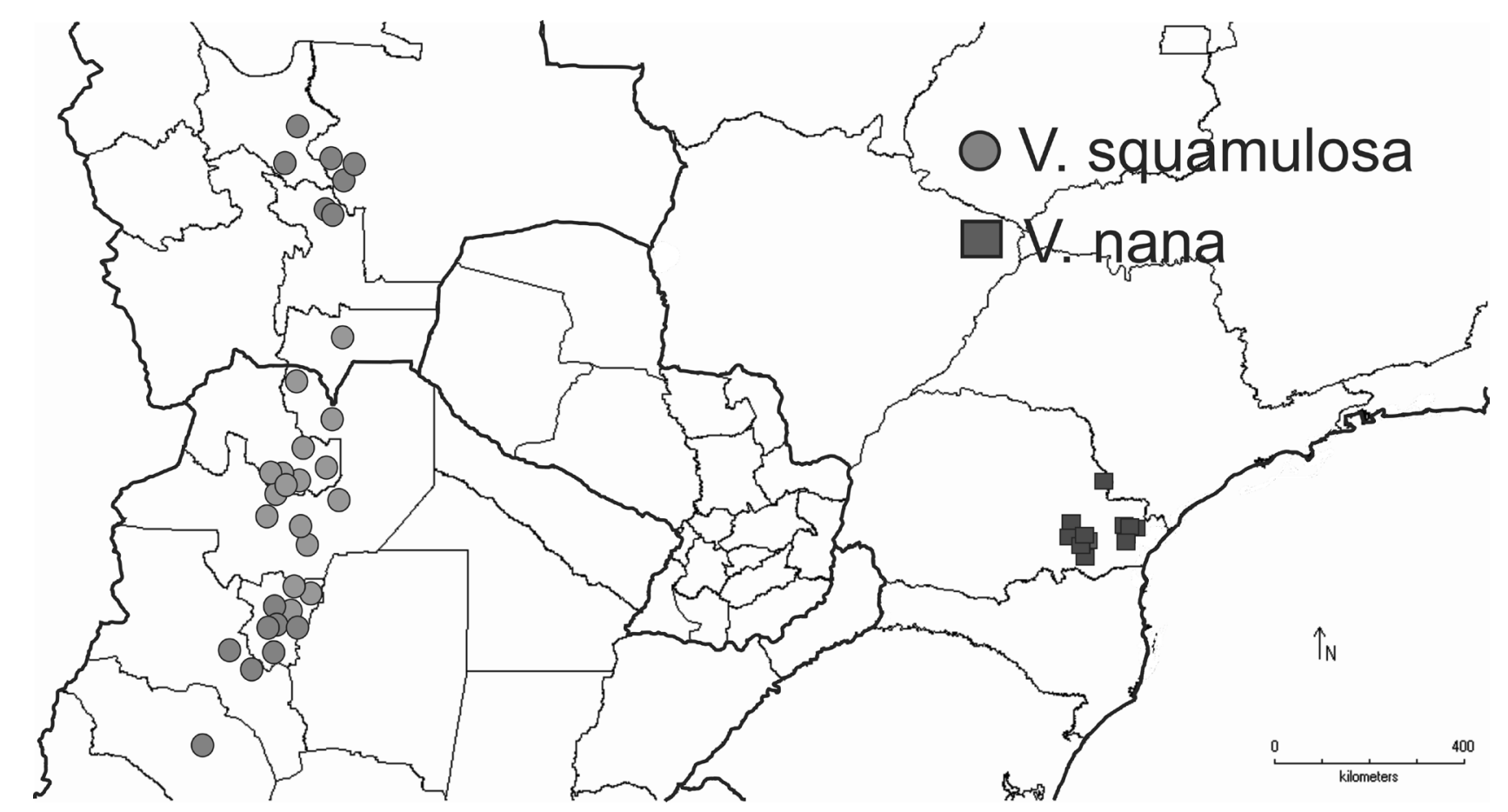

FIGURE 2. Geographic distribution of Vernonanthura squamulosa and V. nana.

\section{Key to the species related to Vernonanthura nana}

1. Leaves glabrous or with small appressed trichomes below. Involucre $5-8 \mathrm{~mm}$ long …....................................................

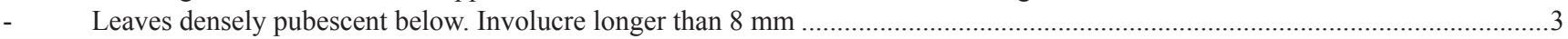

2. Subshrub less than $50 \mathrm{~cm}$ high. Leaves obovate or obovate-oblong, glabrous ............................................................. $\boldsymbol{V} . \boldsymbol{n a n a}$

- $\quad$ Shrub 80-300 cm high. Leaves oblong-lanceolate to lanceolate, with small appressed trichomes ......................... V. montevidensis

3. Leaves tomentose below. Involucre turbinate, $9-11 \mathrm{~mm}$ long ............................................................................ squamulosa

- $\quad$ Leaves lanate below. Involucre urceolate-campanulate, $12-17 \mathrm{~mm}$ long .....................................................................

\section{References}

Bentham, G. (1873) Compositae. In: Bentham, G. \& Hooker, J.D. (Eds.) Genera Plantarum, vol. 2(1). Reeve \& Co., London, pp. 163533.

Bremer, K. (1994) Asteraceae. Cladistics and classification. Timber Press, Portland, pp. 1-752.

Cabrera, A.L. (1944) Vernonias Argentinas (Compositae). Darwiniana 6 (3): 19-379.

Cassini, A.H.G. (1819) Suite du sixième mémoire sur la famille des synanthérées, contenant les caractères des tribus. Journal de Physique, de Chimie, d'Histoire Naturelle et des Arts 88: 189-204.

Dematteis, M. (2002) Cytotaxonomic analysis of South American species of Vernonia (Vernonieae: Asteraceae). Botanical Journal of the Linnean Society 139: 401-408. https://doi.org/10.1046/j.1095-8339.2002.00074.x

Dematteis, M. (2006) Vernonanthura warmingiana (Asteraceae: Vernonieae), a new species from Brazil. Brittonia 58 (2): $182-188$. https://doi.org/10.1663/0007-196X(2006)58[182:VWAVAN]2.0.CO;2

Dematteis, M. \& Fernández, A. (1998) Karyotypes of seven South American species of Vernonia (Asteraceae). Cytologia 63: 323-328. https://doi.org/10.1508/cytologia.63.323

Grisebach, A. (1879) Symbolae ad Floram Argentinam. Abhandlungen der Königlichen Gesellschaft der Wissenschaften zu Göttingen 24: $1-345$.

Hooker, W.J. \& Arnott, G.A.W. (1836) Contributions towards a flora of South America and the islands of the Pacific. Companion to the Botanical Magazine 2: 41-53.

Keeley, S.C. \& Robinson, H. (2009) Vernonieae. In: Funk, V.A., Susanna, A., Stuessy, T.F. \& Bayer, R.J. (Eds.) Systematics, evolution and biogeography of Compositae. International Association for Plant Taxonomy, Vienna, pp. 439-469. 
Robinson, H. (1992) A new genus Vernonanthura (Vernonieae: Asteraceae). Phytologia 73: 65-76. https://doi.org/10.5962/bhl.part.16736

Robinson, H. (1995) New combinations and new species in American Vernonieae (Asteraceae). Phytologia 78 (5): $384-399$. https://doi.org/10.5962/bhl.part.10065

Robinson, H. (1999) Generic and subtribal classification of American Vernonieae. Smithsonian Contributions to Botany 89: 1-116. https://doi.org/10.5479/si.0081024X.89

Robinson, H. (2007) Tribe Vernonieae. In: Kubitzki, K. (Ed.) The families and genera of vascular plants, vol. 8. Springer-Verlag, Berlin, Heidelberg \& New York, pp. 165-192.

Schreber, J.C.D. von (1791) Genera plantarum, 8th ed., vol. 2. Varrentrapp \& Wenner, Frankfurt, pp. 381-872.

Sprengel, C.P.J. (1826) Systema vegetabilium, vol. 3. Sumtibus Librariae Dieterichianae, Göttingen, 414 pp.

Stutts, J.G. (1988) Taxonomic revision of Vernonia subsect. Chamaedrys (Compositae: Vernonieae). Rhodora 90: 37-99.

Vega, A.J. \& Dematteis, M. (2010) The transfer of Vernonia perangusta Malme to the genus Vernonanthura (Vernonieae, Asteraceae) and the correct name for Vernonanthura phosphorica (Vell.) H. Rob. Phytotaxa 8: 46-50.

https://doi.org/10.11646/phytotaxa.8.1.5

Vega, A.J. \& Dematteis, M. (2011a) Nuevas combinaciones y tipificaciones en el género Vernonanthura (Vernonieae, Asteraceae). Boletín de la Sociedad Argentina de Botánica 46: 369-374.

Vega, A.J. \& Dematteis, M. (2011b) Pollen morphology of some species of the genus Vernonanthura (Asteraceae, Vernonieae). Palynology 35: 94-102.

https://doi.org/10.1080/01916122.2010.549290

Vega, A.J. \& Dematteis, M. (2012) Chromosome studies of some species of Vernonanthura and Vernonia (Asteraceae, Vernonieae). Caryología 65: 271-275.

https://doi.org/10.1080/00087114.2012.752912

Vellozo, J.M. da C. (1825 [1829]) Florae fluminensis. Typographia nationali, Rio de Janeiro, 352 pp. 